The Japanese Journal of Animal Psychology, 50, 1, 41-48 (2000)

Original Article

\title{
Relative memory load as a determinant of learning process in reward serial learning by rats
}

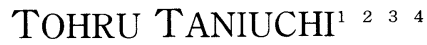 \\ Department of Psychology, University of Tsukuba, Tsukuba, Ibaragi, 305-8572.
}

\begin{abstract}
16 rats were tested in a runway to examine effects of memory load required for item association learning and serial position learning as a determinant of learning process in reward serial learning. Four groups of rats were trained with series A-A-B-N (Group 1-S), A-A-B-N and B-B-A-N (Group 2-S), A-A-A-A-A-B-N (Group 1-L), or A-A-A-A-A-B-N and B-B-B-B-B-A-N (Group 2-L) during acquisition. $\mathrm{N}$ symbolizes nonreward, and $\mathrm{A}$ and $\mathrm{B}$ were reward items which differed in quality. There were two test phases. In the item change test, the last part of the original series A-B-N and B-A-N was changed to A-A-N and B-B-N, respectively. In the position change test, the series were shortened by deleting their first items. Through these tests, it was suggested that Group 2-S learned serial position of nonreward, whereas the other three groups formed item associations between nonreward and preceding items. These results indicate that rats learn serial position of an item when both memory load for item association learning is high and for position learning is low, but in case memory load for position learning is high, they form item associations combining multiple item memory as discriminative cues for nonreward even under conditions where simple item cues are not available as signals. This finding suggests that rats can extract multiple types of information simultaneously from a series and can make decisions about learning strategies by comparing memory load required for the strategies.
\end{abstract}

Key words : rats, serial learning, associative learning, serial position, runway.

Item information and position information have been regarded as major sources for discriminative cues in serial learning in humans (e. g., Slamecka, 1985). The item view suggests that stimuli derived from earlier items supply

1 Research Fellow of Japan Society for Promotion of Science

2 I would like to thank Dr. J. Komaki of Kanazawa University and Dr. J. Makino of University of Tsukuba for their comments on an earlier version of the article. I also wish to thank Dr. D. Erickson of National Institute of Multimedia Education for her comments on this paper and helpful advice on English expressions.

3 Reprint request e-mail : tohruta@kenroku.kanazawa-u.ac.jp

4 Present address: Faculty of Letters, Kanazawa University, Kakuma-machi, Kanazawa, 920-1192 (Tel: 076-264-5316) discriminative cues for later items, whereas the position view emphasize that serial position information guides discriminative responding to items.

Serial learning in rats has been studied in the form of reward serial learning in which items of a series often vary in magnitude of $0.045 \mathrm{~g}$ food pellets (e. g., Hulse \& Dorsky, 1977) or differ in quality (e. g., Capaldi \& Miller, 1988b). These items are given in the goal box of a straight runway in a fixed order on consecutive runs. Learning is indexed by slower running to smaller rewards, especially to nonreward, and faster running to larger rewards.

Although there was a study that suggested position learning is the dominant learning process in rats' reward serial learning (Roitblat, Pologe, \& Scopatz, 1983), later studies have demonstrated that rats mainly form item 
associations or respond to other types of item-derived information, and do not learn serial position of items when item cues are available (e. g., Capaldi, Alptekin, Miller, Birmingham, 1997; Haggbloom, 1985; Taniuchi, 1998a).

However, it has been demonstrated that rats can, to some extent, count the number of reward events to anticipate following nonreward when simple item cues were not available (e. g., Burns \& Gordon, 1988; Burns \& Nesbitt, 1990; Burns, Nesbitt, Cribb, \& Hulbert, 1990; Burns \& Sanders, 1987; Capaldi \& Miller, 1988a). Since serial position information can be supplied from the number of rewards or trials, their counting ability seems to afford a basic capacity for serial position learning.

What, then, is a determinant of the learning process? As mentioned above, one of them is availability of simple item cue. But it is not clear whether learning process is merely determined by availability of the dominant item cue, or rats can extract multiple types of information from a series and make decisions based on relative memory load of the information. The purpose of this report is to examine whether the decision of learning processes between item association learning and serial position learning is influenced by relative memory load required for each of the learning strategies.

A $2 \times 2$ factorial design produced four groups. The first factor was Item load. Groups in single series condition were trained with a single series, whereas groups in double series condition received two series. The second factor was Position load. Groups in short series condition were presented with four item series (S: short series), whereas groups in long series condition received seven item series (L: long series). Therefore, series used in training were A-A-B-N for Group 1-S, A-A-B-N and B-B-A-N for Group 2-S, A-A-A-A-A-B-N for Group 1-L, and A-A-A-A-A-B-N and B-B-B-B-B-A-N for Group 2 - $\mathrm{L}$, where the first digits designates the number of series given to the groups (item load), and the letters after the hyphen designate the length of the series (position load). A, B, and N symbolize two kinds of reward and nonreward items, respectively.

It was expected that Groups $1-\mathrm{S}$ and $1-\mathrm{L}$ learned their series based on item association because the simple item cue $B$ was available for signal of nonreward in their series. On the other hand, in Groups 2-S and 2-L, simple item cues were not available for signals of nonreward because $\mathrm{A}$ and $\mathrm{B}$ signaled both reward item and nonreward item in either of the series. Since it has been demonstrated that rats can combine multiple item memory as discriminative cues for later items (e. g., Capaldi \& Miller, 1988b; Capaldi, Nawrocki, \& Verry, 1983; Capaldi \& Verry, 1981; Taniuchi, 1997a), rats in the double series condition could anticipate nonreward by combining, at least, the preceding two item cues A-B and B-A. But such a type of item association learning requires greater memory load than simple item association learning. Then, under the double series condition, rats might learn serial position of the nonreward instead of forming item associations when the series was short and memory load for serial position learning was low but not when the series was long and memory load was high for serial position learning. Therefore, it was predicted that Groups 1-S, 1-L, and 2-L learned their series based on item association learning, whereas Group 2-S might learn their series through serial position learning.

Following the acquisition training, there were two test phases where item cues or position cues were changed. In the item change test, series were changed from the original series to A-A-A-N, A-A-A-A-A-A-N, B-B-B-N, and B-B-B-B-B-B-N, respectively. In the position change test, series were shortened from the original ones to A-B-N, A-A-A-A-B-N, B-A-N, and B-B-B-B-A-N, respectively. According to the prediction described above, it was expected that item change disrupts the anticipation of nonreward in Groups 1-S, 1-L, and 2-L but not in Group 2-S, and position change disrupts the anticipation of nonreward only in Group 2-S but not in Groups 1-S, 1-L, and 2-L.

\section{Method}

\section{Subjects}

The subjects were 16 Sprague Dawley rats approximately 180 days old. These animals had experience of being shaped to press a lever of an 
Taniuchi : Relative memory load as a determinant of learning process in reward serial learning by rats

operant chamber about 90 days before the experiment.

\section{Apparatus}

The apparatus was an enclosed straight runway, $162.0 \mathrm{~cm}$ long, $10.0 \mathrm{~cm}$ wide, $11.0 \mathrm{~cm}$ high, and covered by wire mesh on hinged frames. The start box and the goal box were $30.0 \mathrm{~cm}$ and 40.0 $\mathrm{cm}$ long respectively, and could be closed off by guillotine doors. These boxes were painted brown and the remaining area of the runway was painted flat black. A digital timer started when the start door was raised and stopped when a photobeam located $25.0 \mathrm{~cm}$ into the goal box was interrupted by a rat. The goal door was lowered when the photobeam was interrupted, and confined the rat to the goal box. Rewards could be placed in a food cup, $5.0 \mathrm{~cm}$ in diameter and $0.8 \mathrm{~cm}$ in depth, located at the end of the goal box. Rewards were $0.045 \mathrm{~g}$ food pellets and puffed sweetened rice.

\section{Pretraining}

Rats were randomly assigned to four groups. During the first twelve days, rats were handled for $1 \mathrm{~min}$ per day, and reduced to $85 \%$ of ad-lib body weight by food deprivation. On Days 13-14, each rat was given $10 \mathrm{~min}$ of exploration of the runway with all the doors open, and permitted to eat six food pellets and three grains of puffed sweetened rice scattered over the floor of the runway. On Day 15, rats were confined to the goal box with the door lowered, and given six food pellets and three grains of puffed sweetened rice from the food cup.

\section{Experimental training}

Experimental training consisted of four phases: acquisition, an item change test, recovery, and a position change test. Acquisition began on Day 16 and continued for 28 days. Two groups of the short series condition received the short four item series, whereas the other two groups of the long series condition were given the long seven item series. One of the two groups in each of the short series and the long series condition received a single series terminating A-B-N throughout acquisition. In this single series condition, item $B$ was available as a cue for following nonreward. N symbolizes nonreward. $\mathrm{A}$ and $\mathrm{B}$ were either three $0.045 \mathrm{~g}$ food pellets or a grain of puffed sweetened rice and counterbalanced between subjects. The other groups in the short series and the long series condition received two series terminating A-B-N and B-A-N, concurrently. In this double series condition, the simple item cue A and B could not be available as a cue for nonreward. Thus, the four groups were designed 1-S, 2-S, 1-L, and 2-L, where the first digits designates the number of series given to the groups, and the letters after the hyphen designate the length of the series. The series given to these groups were A-A-B-N for Group 1-S, A-A-B-N and B-B-A-N for Group 2-S, A-A-A-A-A-B-N for Group 1-L, and A-A-A-A-A-B-N and B-B-B-B-B-A-N for Group 2-L. Groups 1-S and 1-L received four presentations of the single series per day. Groups 2-S and 2-L received two presentations of the two series per day. For groups in the double series condition, the order of presenting the series was ABBA on odd days and BAAB on even days for half the subjects ( $A$ and $B$ represent the first items of the series), and $\mathrm{BAAB}$ on odd days and ABBA on even days for the remaining half subjects.

On Days 44 and 45, the item change test was conducted. In this test, the last part of the original series A-B-N or B-A-N was changed to A-A-N or $\mathrm{B}-\mathrm{B}-\mathrm{N}$, respectively, thus the series were A-A-A-N, A-A-A-A-A-A-N, B-B-B-N, and B-B-B-B-B-B-N. All other aspects of the procedures were as in the acquisition phase.

Days 46 to 51 were the recovery training phase. The procedures in this phase were identical to those in the acquisition phase.

On Days 52 and 53, the position change test was conducted. In this phase, first items of the original series were deleted and series produced were A-B-N, A-A-A-A-B-N, B-A-N, and B-B-B-B-A-N. All other aspects of the procedures were as in the acquisition phase.

Rats were brought into the experimental room and trained in squads of eight, two rats from each group. A run began with placement of the rat in the start box, and the start door was raised $3 \mathrm{sec}$ later. On rewarded runs, the rat was removed to the holding cage after the reward was consumed. On nonrewarded runs, the rat was confined to the goal box for $20 \mathrm{sec}$, then removed to the waiting cage. If a rat failed to complete a run in $60 \mathrm{sec}$, it 
was removed to the goal box by the experimenter. Interrun interval was $20 \mathrm{sec}$, and interseries interval was 20-30 min. During the interseries interval for a rat, the remaining rats received the series presentation.

\section{Results}

\section{Acquisition}

Figure 1 shows running speeds for each of the groups to first rewarded items and last nonreward items during the acquisition phase in blocks of two days. An analysis of variance was applied to these data. Main effects and interactions of Item $[F(1,12)=9.69, p<.01]$, Block $[F(13,156)=12.15, p<.01]$, Item load $\times$ Item $[F(1,12)=6.67, p<.03]$, Item load $\times$ Block $[F$ $(13,156)=4.46, p<.01]$, and Item $\times$ Block $[F(13$, $156)=21.96, p<.01]$ were significant. Simple effect tests produced the significant effect of Item load at nonreward item $[F(1,24)=9.26, p<.01]$ but not at first item $(F<1)$. These results indicate that the single series condition more rapidly developed slow running to nonreward than the double series condition.

\section{Item change test}

Figure 2 shows running speeds for each of the groups to their series averaged over the last two days of the acquisition phase and both days of the item change test phase. An analysis of variance using data of first items and last nonreward items indicated significant main effects and interactions of Phase $[F(1,12)=53.52$, $p<.01]$, Item $[F(1,12)=31.57, p<.01]$, Item load $\times$ Phase $[F(1,12)=15.19, p<.01]$, Position load $\times$ Phase $[F(1,12)=5.07, p<.05]$, Item $\times$ Phase $[F(1,12)=196.46, p<.01]$, Item load $\times$ Item $\times$ Phase $[F(1,12)=59.51, p<.01]$, and Item load $\times$ Position load $\times$ Item $\times$ Phase $[F(1,12)=5.23$, $p<.05]$. Simple main effect tests conducted on the higher order interaction of Item load $\times$ Position load $\times$ Item $\times$ Phase indicated reliable main effect of Phase in Groups 1-S $[F(1,24)=$ $94.40, p<.01], 1-\mathrm{L} \quad[F(1,24)=115.19, p<.01]$, and 2-L $[F(1,24)=29.04, p<.01]$, but not in $2-\mathrm{S}$ $[F<1]$. These results indicate that the anticipation of nonreward in Groups 1-S, 1-L, and 2-L was deteriorated by item change, but Group 2 -S maintained their anticipation of nonreward.

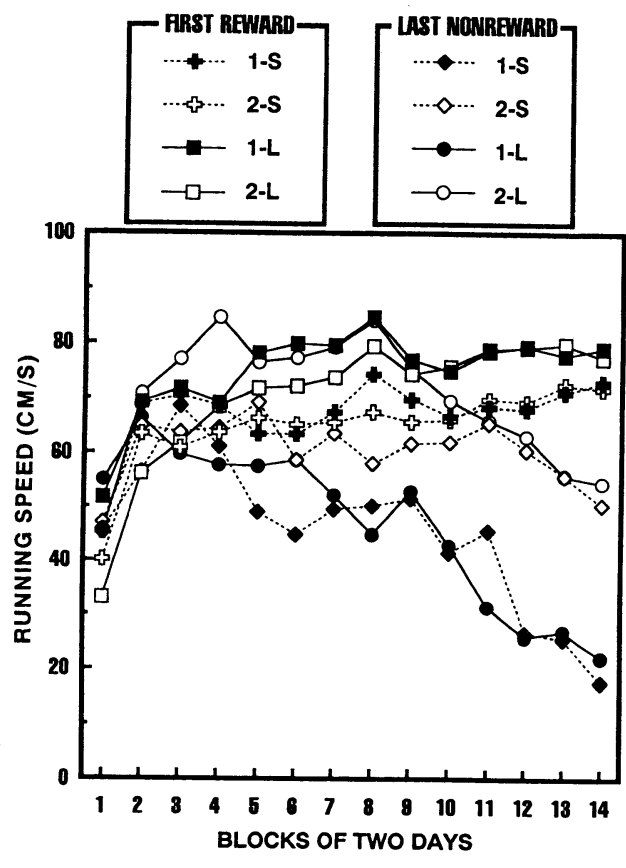

Figure 1. Mean running speeds of Groups 1-S, 2-S, $1-\mathrm{L}$, and 2-L to first reward items and last nonreward items of their series during acquisition phase. Series were A-A-B-N for Group 1-S, A-A-B-N and B-B-A-N for Group 2-S, A-A-A-A-A-B-N for Group 1-L, and A-A-A-A-A-B-N and B-B-B-B-B-A-N for Group 2-L. N symbolizes nonreward, and $\mathrm{A}$ and $\mathrm{B}$ were reward items which differed in quality.

\section{Position change test}

Figure 3 shows running speeds for each of the groups to their series averaged over the last two days of the recovery phase and both days of the position change test phase. An analysis of variance using data of first items and last nonreward items produced significant main effects and interactions of Item load $[F(1,12)=$ $16.80, p<.01]$, Phase $[F(1,12)=9.33, p<.01]$, Item $[F(1,12)=73.93, p<.01]$, Item load $\times$ Item $[F(1,12)=9.41, p<.01]$, Phase $\times$ Item $[F(1,12)=$ 28.57, $p<.01]$, Position Load $\times$ Phase $\times$ Item $[F$ $(1,12)=9.98, p<.01]$. Simple effect tests on the interaction of Position Load $\times$ Phase $\times$ Item indicate that the anticipation of nonreward was deteriorated in the short series condition $[F(1$, $24)=37.63, p<.01]$ but not in the long series condition $[F(1,24)=2.49]$. As an additional analysis, simple main effects of Item in each of the groups at the position change test phase were 


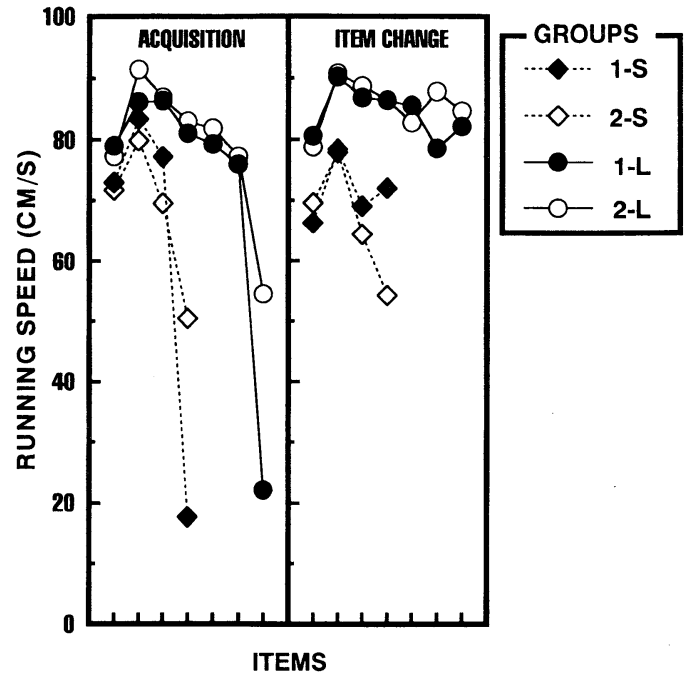

Figure 2. Mean running speeds of Groups 1-S, 2-S, 1-L, and 2-L to their series averaged over the last two days of acquisition phase and both days of item change test phase. In the item change test, the last part of the original series A-B-N and B-A-N were changed to A-A-N and B-B-N, respectively.

examined. Contingent with position change, anticipation of nonreward was eliminated in Group 2-S $[F<1]$, but maintained in the other three groups $[F s(1,24)=19.57,35.43,9.58, p s<.01$, for Groups 1-S, 1-L, 2-L, respectively].

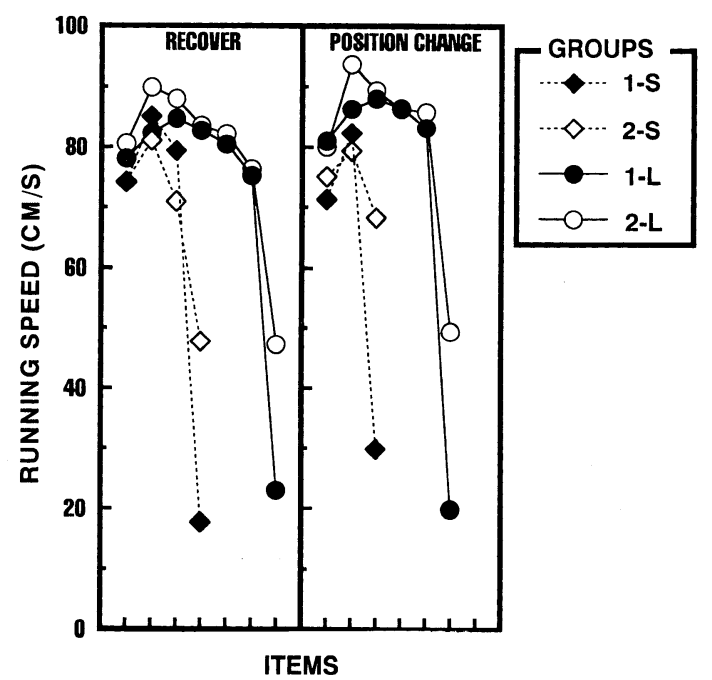

Figure 3. Mean running speeds of Groups 1-S, 2-S, 1-L, and 2-L to their series averaged over the last two days of recovery phase and both days of position change test phase. In the position change test, the series were shortened by deleting their first items.

\section{Discussion}

Several studies have shown that rats learn item association but not serial position of an item when simple item cues are available (e. g., Burns, Hulbert, \& Cribb, 1990; Capaldi \& Miller, 1988a; Haggbloom, 1985; Mizuhara 1993). Also, many serial pattern learning studies have shown that structures or item discriminability of preceding items affect anticipation of later nonreward items of series in which nonrewards are in equal serial position (e. g., Capaldi \& Molina, 1979; Hulse \& Dorsky, 1977; Taniuchi, 1992). Furthermore, adding position information to item cues does not facilitate learning (e. g., Capaldi et al., 1997; Capaldi \& Miller, 1988b). These findings could be interpreted as evidence which show dominance of item cues in reward serial learning by rats.

In the present study, Groups 1-S and 1-L, in which simple item cues were available as signals for nonreward, learned item associations regardless of the memory load for position cues. These results are consistent with previous work which showed dominance of item cue.

The results in the item change test and the position change test in the present investigation consistently indicated that Group 2-S learned serial position of nonreward but Group 2-L formed item associations between nonreward and preceding items. Since information load or validity of item cues were equal between these groups, it could be concluded that learning processes are influenced by memory load of position cues. This is the first time it has been demonstrated that a determinant of learning processes in rats' serial learning is not absolute information load or saliency of a cue but rather the relative memory load among cues. This suggests that rats can extract multiple types of information simultaneously from a series and can make decisions about learning strategies by comparing memory load required for the strategies.

There are several types of information which have been said to be able to mediate serial position learning, such as the number of trials or reward events, time elapsing from the start of a series, amount of time confined to the goal box. The present investigation could not identify the 
information which mediated serial position learning in Group 2-S, because the number cues and the time cues were confounded in the condition. However, some studies showed that rats utilized the number of reward events rather than time cues or number of trials when these cues were available simultaneously. For example, Capaldi \& Miller (1988a) trained rats with 10-10-0 series (digits represent number of $0.045 \mathrm{~g}$ food pellets) and then shifted the series to 0-10-10-0 and also changed the confinement time to the goal box from $15 \mathrm{sec}$ to $30 \mathrm{sec}$. The shifts in the series and confinement time did not deteriorate the anticipation of 0 pellet. Contingent with such shifts, the number of trials and the two kinds of time cues become invalid but the number of reward events was still valid. Therefore, these results indicate that rats mainly utilize the number of reward events but not that of trials or time cues when these cues were available simultaneously. Thus, there seems to be a strong possibility that serial position learning in Group 2-S was mediated by information about the number of reward events rather than that of trials, or time cues. However, Mizuhara (1993) reported a large deterioration of the 0 pellet anticipation contingent with series shift from 5-5-0 to 0-5-5-0. Such a finding is somewhat at variance with those of Capaldi \& Miller (1988a), and suggests the possibility that rats utilize to some extent the number of trials or time cues. Learning processes of serial position learning and the variables which affect them remain open questions.

Although results in the present investigation clearly show that the learning process is determined by relative memory load of the cues, we could not predict exactly which cues would be used, because we have no basis to compare the load of different types of information, such as serial position and item memory. It can only be conjectured after the fact. In spite of such a difficulty, critical functions of memory load on decision of learning strategy has been shown in a variety of animal learning situations. For example, the number of stimulus sets is one of the determinants of learning strategy in matching to sample task or same-different discrimination learning by many animal species (e. g., Roitblat \& von Fersen, 1992). Also, in rats' serial learning, it was proposed that rats learn rule structure of series instead of item association depending on the memory load to be reduced (Hulse, 1980). Support for the hypothesis comes from several studies (Fountain, Schenk, \& Annau, 1985; Taniuchi, 1992, 1997b). Thus, memory load must be one of the important concepts in explaining determinants of learning process in various learning phenomena.

Recently, many experimental methods of reward serial learning have been developed (Taniuchi, 1998b). In reward serial learning on the radial maze, some hypotheses regard position information as a major cue for reward anticipation behavior (e. g., Neath \& Capaldi, 1996; Wathen \& Roberts, 1994). In this learning situation, reward series were assigned to arms of the maze and four or eight series were presented concurrently. Under such a condition, when item association learning possibly required high memory load, position information might be relatively valid cues. The memory load approach may answer why dominant cues are different in the runway and the radial maze situations.

Serial position learning is shown to be the dominant learning process in monkey serial learning where animals are required to respond in a particular order to simultaneously presented visual items (e. g., D'Amato \& Colombo, 1988). We can not compare this finding directly with rats' reward serial learning since there are great differences in item material and presentation method. However, the suggested discrepancy in learning process of serial learning between rats and monkeys is very intriguing because it suggests different cognitive processing among species. Examination of the generality of findings in rats' reward serial learning studies across various item materials and experimental paradigms, especially those have been used in studies with other animal species, is a very important avenue for future research into better understanding animal cognition.

\section{References}

Burns, R. A., \& Gordon, W. U. 1988 Some further observations on serial enumeration and 
Taniuchi : Relative memory load as a determinant of learning process in reward serial learning by rats

categorical flexibility. Animal Learning \& Behavior, 16, 425-428.

Burns, R. A., Hulbert, L. G., \& Cribb, D. 1990 A test for order relevance in a three-element serial learning task. The Journal of General Psychology, 117, 91-98.

Burns, R. A., \& Nesbitt, F. A. 1990 A test for S-S associations in a conditional counting task. Bulletin of the Psychonomic Society, 28, 441444.

Burns, R. A., Nesbitt, F. A., Cribb, D., \& Hulbert, L. G. 1990 Conditional discrimination of series containing either two or three rewarded trials. The Journal of General Psychology, 117, 161-170.

Burns, R. A., \& Sanders, R. E. 1987 Concurrent counting of two and three events in a serial anticipation paradigm. Bulletin of the Psychonomic Society, 25, 479-481.

Capaldi, E. J., Alptekin, S., Miller, D. J., \& Birmingham, K. M. 1997 Is discriminative responding in reward outcome serial learning mediated by item memories or by position cues. Learning and Motivation, 28, 153-169.

Capaldi, E. J., \& Miller, D. J. 1988a Counting in rats: Its functional significance and the independent cognitive processes that constitute it. Journal of Experimental Psychology: Animal Behavior Processes, 14, 317.

Capaldi, E. J., \& Miller, D. J. 1988b The rat's simultaneous anticipation of remote events and current events can be sustained by event memories alone. Animal Learning \& Behavior, 16, 1-7.

Capaldi, E. J., Nawrocki, T. M., \& Verry, D. R. 1983 The nature of anticipation: An inter- and intraevent process. Animal Learning \& Behavior, 11, 193-198.

Capaldi, E. J., \& Verry, D. R. 1981 Serial order anticipation learning in rats: Memory for multiple hedonic events and their order. Animal Learning \& Behavior, 9, 441-453.

D'Amato, M. R., \& Colombo, M. 1988 Representation of serial order in monkeys (Cebus apella). Journal of Experimental Psychology: Animal Behavior Processes, 14, 131-139.
Fountain, S. B., Schenk, D. E., \& Annau, Z. 1985 Serial - pattern - learning processes dissociated by trimethyltin exposure in rats. Physiological Psychology, 13, 53-62.

Haggbloom, S. J. 1985 Serial learning and transfer in rats: Effects of changes in stimulus-stimulus associations, pattern structure, and serial position information. Animal Learning \& Behavior, 13, 370-374.

Hulse, S. H. 1980 The case of the missing rule: Memory for reward vs. formal structure in serial pattern learning by rats. Animal Learning \& Behavior, 8, 689-690.

Hulse, S. H., \& Dorsky, N. P. 1977 Structural complexity as a determinant of serial pattern learning. Learning and Motivation, 8, 488-506.

Mizuhara, Y. 1993 Counting, the amount of reward, interitem associations, and serial position in serial learning in rats. Hiroshima forum for Psychology, 15, 1-10.

Neath, I., \& Capaldi, E. J. 1996 A "random-walk" simulation model of multiple-pattern learning in a radial-arm maze. Animal Learning \& Behavior, 24, 206-210.

Roitblat, H. L. \& Fersen, L. von. 1992 Comparative cognition: Representation and processes in learning and memory. Annual Review of Psychology, 43, 671-710.

Roitblat, H. L., Pologe, B., \& Scopatz, R. A. 1983 The representation of items in serial position. Animal Learning \& Behavior, 11, 489-198.

Slamecka, N. J. 1985 Ebbinghaus: Some associations. Journal of Experimental Psychology: Learning, Memory, and Cognitions, 11, 414-435.

Taniuchi, T. 1992 Effects of interrun interval and shift of reward sequence on serial pattern learning in rats. The Japanese Journal of Animal Psychology, 42, 77-86. (In Japanese with English Abstract)

Taniuchi, T. 1997a Effects of item-arrangement on serial pattern learning and extinction in rats. The Japanese Journal of Psychology, 68, 255-263. (In Japanese with English Abstract)

Taniuchi, T. 1997b Effects of interrun interval shift and pattern length on serial pattern learning in rats. The Japanese Journal of Animal Psychology, 47, 27-35. (In Japanese 
with English Abstract)

Taniuchi, T. 1998a Concurrent learning of multiple serial patterns and transfer in rats. The Japanese Journal of Animal Psychology, 48, 11-22. (In Japanese with English Abstract) Taniuchi, T. 1998b Basic evidence and recent developments of serial pattern learning in rats. Japanese Psychological review, 41, 392-407. (In Japanese with English Abstract)

Wathen, C. N., \& Roberts, W. A. 1994 Multiple-pattern learning by rats on an eight-arm radial maze. Animal Learning \& Behavior, 22, 155-164.

(Received Oct. 2, 1999 ; accepted Feb. 2, 2000) 